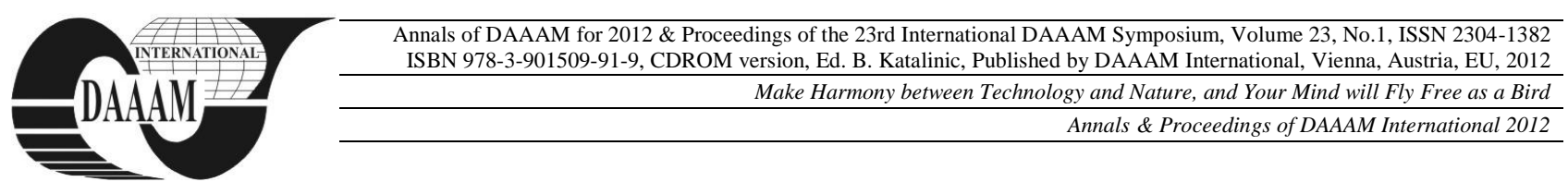

\title{
DEVICE FOR WIRELESS LIGHT INTENSITY REGULATION WITH IR PROTOCOL
}

\author{
LJUTIC, S[anjin]; ZENZEROVIC, P[aolo] \& VRANKIC, M[iroslav]
}

\begin{abstract}
This paper presents a device whose main purpose is wireless light intensity regulation with IR protocols. The concept and design for realization of such a device is presented in this paper. All assumptions and solutions have been tested through computer simulation and complete hardware implementation. . Besides that it's main purpose of the device is presented as an educational technology tool for learning basic communication protocols at university of digital electronics classes.

Keywords: wireless light intensity regulation, infra-red (IR) protocols, IR receiver, universal IR light dimmer, educational technology
\end{abstract}

\section{INTRODUCTION}

Today light intensity regulation systems are present at more and more industrial, working and home facilities. The importance of light intensity regulation is pointed out by many authors $[1,6]$. The main benefits include minimized power consumption, prolonged life span of light bodies, possibility of adaptive lighting for ergonomic purposes, etc. As such systems are being more widely used it is reasonable to suppose specific knowledge for such systems will be required for electrical engineers in more and more companies, that are working in this field of electrical engineering. Therefore, a fully functional light dimmer is presented here, not only as a regulating device but also as an educational technology tool.

The author's main goal was the realization of a device that serves as a light regulation system that would be capable of getting commands wirelessly throught infrared channels. The main problem was how to design a device that can be used for regulation of light intensity with infrared protocol communication; with any type of remote control, and would be capable of regulation the light intensity of any type of light source.

The idea was to design a device which will be used at homes for light regulation and the user can regulate the light intensitiy with a remote control commonly used for controlling TV, VCR or some other device controlled with IR remote control.

For the realization of this device (universal IR light dimmer), certain questions had to be addressed:

- How to develop the light dimmer with as few electronics parts as possible to make it as cheap as possible

- What type of output should the dimmer provide to best fit a general purpose usage
- Which IR communication protocols are most commonly used

- Which is the simplest way to decode the most used infrared protocols

\section{AVAILABLE SOLUTIONS}

Today there are several available types of wireless light intensity regulation devices with IR protocols.

\subsection{The rheostat dimmer}

Dimmers based on rheostats are inefficient since they dissipate a significant portion of the power rating of the load as heat. They are large and require a lot of air to circulate in order to dissipate the generated heat. Because their dimming effect depend a great deal on the total load applied to each rheostat, the load needs to be matched fairly carefully to the power rating of the rheostat. Finally, as they rely on mechanical control they are slow and it is difficult to change many channels at a time [1].

\subsection{The saltwater dimmer}

An early example of a dimmer includes the salt water dimmer. In saltwater dimmers, there were two metal contacts in a glass beaker. One contact was on the bottom, while the other was able to move up and down. The closer the contacts were to each other, the level of the light was higher. Salt-water dimmers were not efficient due to the evaporation of water and the corrosion of the many metal pieces, therefore those are not used at present [1].

\subsection{The coil-rotation transformer}

The coil - rotation transformer uses a fixed-position electromagnet coil in conjunction with a variableposition coil to vary the voltage in the line by varying the alignment of the two coils. Rotated 90 degrees apart, the secondary coil is affected by two equal but opposite fields from the primary, which effectively cancel each other out and produce no voltage in the secondary.

These coils resembled the standard rotor and stator as used in an electric motor, except that the rotor was held against rotation using brakes and was moved to specific positions using high-torque gearing. Because the rotor did not ever turn a complete revolution, a commutator was not required and long flexible cables could be used on the rotor instead [3].

\subsection{The Autotransformer dimmer}

Variable autotransformers were introduced later after coil-rotation transformers. While they were still nearly as 
large as rheostat dimmers, they were relatively efficient devices. Their voltage output, and so their dimming effect, was independent of the load applied so it was far easier to design the lighting that would be attached to each autotransformer channel. Remote control of the dimmers was still impractical, although some dimmers were equipped with motor drives that could slowly and steadily reduce or increase the brightness of the attached lamps. Variacs are no longer in use for lighting but are used for other applications [3].

\subsection{The Thyristor dimmer}

Thyristor dimmers were introduced to solve some problems experienced often with older types of light dimmers. Thyristor dimmers switch on at an adjustable time (phase angle) after the start of each alternating current half-cycle, thereby altering the voltage waveform applied to lamps and so changing its RMS effective value. Because they switch instead of absorbing part of the voltage supplied, there is very little wasted power. Dimming can be almost instantaneous and is easily controlled by remotely by digital electronics [1]

\subsection{The universal light dimmer}

Nowdays light dimmers are usually controlled remotely by means of various protocols. Universal dimmers are capable of dimming any light source, opposed to the previously mentioned dimmer types. The universal light dimmer does not contain the section with power electronics embedded in the system, but functions as a dimming controller. Most often it's output will be a standard industrial regulating signal like variable DC voltage in the range from 0 to $10 \mathrm{~V}$, or variable $\mathrm{DC}$ current from 4 to $20 \mathrm{~mA}$. This type of dimmer is commonly based a microcontroller and some peripheral others integrated components, like digital to analog converters and amplifiers. Universal light dimmers can also be used for dimming fluorescent tube lights. Dimming the fluorescent tubes requires the usage of a compatible electronic dimming ballast.

Because of all the mentioned advantage the dimmer proposed in this paper will be based on an universal light dimmer model.

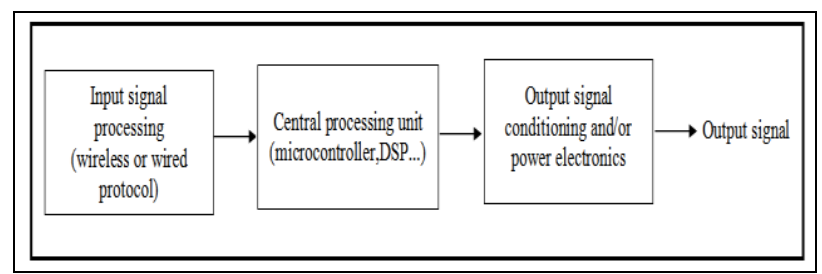

Fig. 1. Block diagram of the universal dimmer

\section{IR PROTOCOLS}

IR protocols are best suited for short distance, low-tomedium data throughput, and wireless communication channels. Each manufacturer uses a different set of protocols, so today we have many types of IR protocols that are currently in use. The most used protocols include the Philips RC5 and RC6 protocols, the Sony SIRC protocol and the NEC protocol. Those three protocols were choosen to decode because of their similarity to other protocols, thus, it would be easy to integrate other protocols in the device if needed.
The RC-5 protocol is a type of Manchester encoded data packet. Manchester data is unique in that a data is signified by a transition in the middle of the bit. A one 1 is received by the IR receiver (after inversion by the IR sensor) as a high-to-low transition, and a zero - 0 as a low-to-high transition. The RC5 IR packet consists of 14 bits: two start bits (S1, S0), one control bit (C), five address bits (A4 to A0), and a six bit command code (C5 to $\mathrm{C} 0$ ). The entire 14-bit packet is received MSB first.

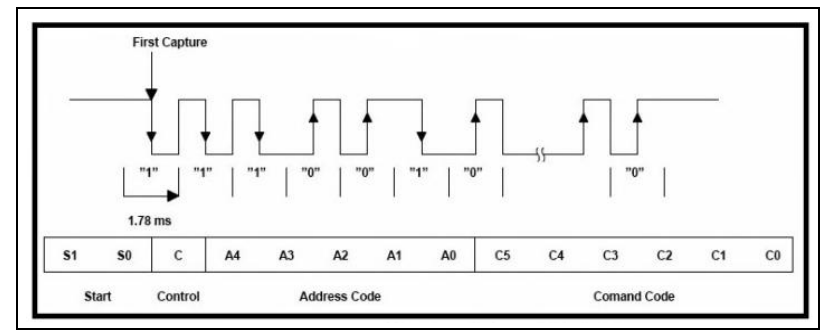

Fig. 2. Inverted RC5 data packet as seen by IR receiver

The start bits are always transmitted as 1 . The control bit toggles whenever a new key is received. The five address bits represent 32 different potential addresses of the equipment for which the packet is intended. The six command bits represent 64 commands that can be transmitted. The bit period for RC5 is $1.78 \mathrm{~ms}$ long, with half of that period high and the other half low. The duration for the complete 14-bit packet is approximately $25 \mathrm{~ms}$ at frequency of $36 \mathrm{kHz}$ [5].

The SONY SIRC (Serial Infra-Red Control) protocol is an infra-red light remote control communication protocol that uses a form of pulse width modulation (PWM) to build serial interface. The most common protocol is a 12-bit interface, but 15-bit and 20_bit version are also available. The SIRC protocol uses a pulse width encoding of the bits. The pulse representing a logical "1" is a $1.2 \mathrm{~ms}$ long burst of the $40 \mathrm{kHz}$ carrier, while the burst width for a logical " 0 " is $0.6 \mathrm{~ms}$ long. All bursts are separated by a $0.6 \mathrm{~ms}$ long space interval. The recommended carrier duty-cycle is $1 / 4$ or $1 / 3$. With this protocol the LSB is transmitted first. The start burst is always $2.4 \mathrm{~ms}$ wide, followed by a standard space of 0.6 ms. Apart from signalling the start of a SIRC message, this start burst is also used to adjust the gain of the IR receiver. Then the 7-bit Command is transmitted, followed by the 5-bit Device address. Commands are repeated every $45 \mathrm{~ms}$ (measured from start to start) for as long as the key on the remote control is held down.

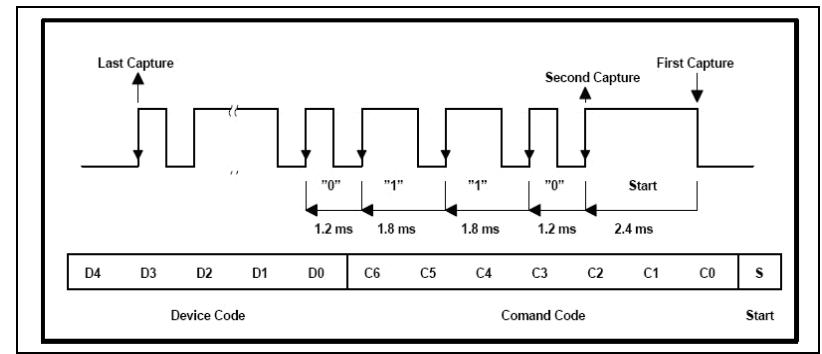

Fig. 3. Sony SIRC data packet

The NEC protocol basic features are: 8 bit address and 8 bit command length, address and command are transmitted twice for reliability. This protocol uses pulse distance encoding of the bits. Each pulse is a 560 us long $38 \mathrm{kHz}$ carrier burst (about 21 cycles). A logical "1" takes $2.25 \mathrm{~ms}$ to transmit, while a logical "0" is only half 
of that, being $1.125 \mathrm{~ms}$. The recommended carrier dutycycle is $1 / 4$ or $1 / 3$.

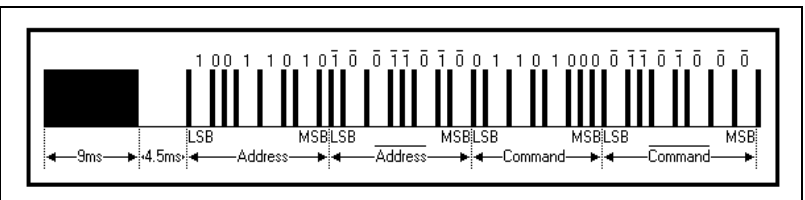

Fig. 4. NEC data packet

A command is transmitted only once, even when the key on the remote control remains pressed. Every $110 \mathrm{~ms}$ a repeat code is transmitted for as long as the key remains down [4].

\section{IMPLEMENTED SOLUTION}

\subsection{Block scheme and working principles}

The implemented solution relies on the topology of a universal light dimming system in order to meet the criteria serve in the introduction chapter. The block scheme is shown in figure 5.

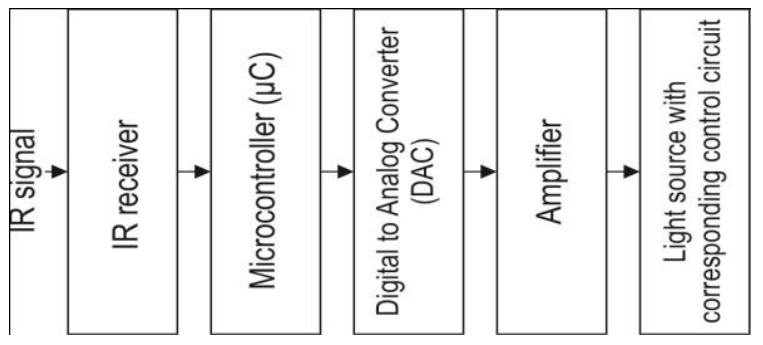

Fig. 5. Block diagram of implemented IR light dimmer

Working principle: the IR signal comes to the IR receiver. The IR receiver is connected with the microcontroller $(\mu \mathrm{C})$. When the IR signal is detected, the microcontroller decodes the command and acts according to its programing. For testing purposes the dimmer was programmed to act as follows: if the key for increasing the volume is pressed, then the light intensity is increases. If the key for decreasing the volume is pressed, then the light intensity lowers. Also, two buttons are available to turn of the light completely and to bring the light to full intensity. When the IR signal is decoded the microcontroller generates the regulation signal for the light emitter. The choosen type of signal is a variable DC voltage signal ranging from 0 to $10 \mathrm{~V}$. The conversion from the digital signal to the analog signal is done by a DAC (digital-analog converter). The microcontroller and the DAC are connected using a serial protocol (I2C). The output from the DAC goes from 0 to $3.3 \mathrm{~V}$ maximum so it should be amplified. The DAC output is wired to a operational amplifier and the voltage is amplified to the specitied range. On the dimmer output, we have DC voltage in the range between 0 and $10 \mathrm{~V}$ control signal for controlling the lamp brightness. In this case $0 \mathrm{~V}$ means that the lamp is off and $10 \mathrm{~V}$ signal means that the lamp is fully on. On the output we can connect any type of light source with the corresponding control circuit for that light source.

The implemented solution is limited to use only with power light dimmer interfaces, it is not possible to use the dimmer itself to drive directly the load. Also, the solution provides only $0-10 \mathrm{~V}$ interface to drive the dimmer interface, more complex protocols were not implemented.

\subsection{Testing results - Voltage output}

The implemented light dimmer voltage output was tested and results are shown in table 1.

\begin{tabular}{|c|c|c|}
\hline $\begin{array}{c}\text { Percentage of } \\
\text { light intensity (\%) }\end{array}$ & $\begin{array}{c}\text { Output voltage } \\
(\mathrm{V})\end{array}$ & $\begin{array}{c}\text { Percent error } \\
(\%)\end{array}$ \\
\hline $0 \%$ & $0 \mathrm{~V}$ & $0 \%$ \\
\hline $10 \%$ & $0.99 \mathrm{~V}$ & $1 \%$ \\
\hline $20 \%$ & $1.99 \mathrm{~V}$ & $0.5 \%$ \\
\hline $30 \%$ & $2.99 \mathrm{~V}$ & $0.333 \%$ \\
\hline $40 \%$ & $3.99 \mathrm{~V}$ & $0.25 \%$ \\
\hline $50 \%$ & $4.99 \mathrm{~V}$ & $0.2 \%$ \\
\hline $60 \%$ & $5.99 \mathrm{~V}$ & $0.166 \%$ \\
\hline $70 \%$ & $6.99 \mathrm{~V}$ & $0.142 \%$ \\
\hline $80 \%$ & $7.99 \mathrm{~V}$ & $0.125 \%$ \\
\hline $90 \%$ & $8.99 \mathrm{~V}$ & $0.111 \%$ \\
\hline $100 \%$ & $9.94 \mathrm{~V}$ & $0.6 \%$ \\
\hline
\end{tabular}

Tab. 1. Voltage characteristic of the implemented dimmer

The used power supply for the dimmer is a common network voltage adapter transformin the voltage from $230 \mathrm{~V}$ AC to $12 \mathrm{~V}$ DC voltage. On the output of the dimmer, we have connected the voltmeter and with it we measured the output voltage depending on the key that is pressed on the remote control (key for increasing or decreasing the light intensity).

\subsection{Testing results - Light output}

The implemented dimmer was tested to regulate the light intensity of a fluo-tube light source. The output of the device was connected to a fluo tube ballast that converted the regulation signal to voltage and frequency adequate to drive the fluo tube. The numerical results are shown in table 2 .

\begin{tabular}{|c|c|c|}
\hline $\begin{array}{c}\text { Percentage of } \\
\text { light intensity } \\
(\%)\end{array}$ & $\begin{array}{c}\text { Output voltage } \\
(\mathrm{V})\end{array}$ & $\begin{array}{c}\text { Light intensity } \\
\text { (Lux) }\end{array}$ \\
\hline $0 \%$ & $0 \mathrm{~V}$ & 0 \\
\hline $10 \%$ & $0.99 \mathrm{~V}$ & 10 \\
\hline $20 \%$ & $2 \mathrm{~V}$ & 24.9 \\
\hline $30 \%$ & $3.01 \mathrm{~V}$ & 57.5 \\
\hline $40 \%$ & $4.02 \mathrm{~V}$ & 114.8 \\
\hline $50 \%$ & $5.03 \mathrm{~V}$ & 161.2 \\
\hline $60 \%$ & $6.04 \mathrm{~V}$ & 196 \\
\hline $70 \%$ & $7.04 \mathrm{~V}$ & 219 \\
\hline $80 \%$ & $8.05 \mathrm{~V}$ & 245 \\
\hline $90 \%$ & $9.04 \mathrm{~V}$ & 275 \\
\hline $100 \%$ & $10.04 \mathrm{~V}$ & 286 \\
\hline
\end{tabular}

Tab. 2. Light intensity regulation results

The measurements of light intensity were taken by a manual lux-meter placed in line with the fluo tube, measuring the intensity of the light being reflected from a white cealing approximately two meters above the fluo lamp and lux-meter. The resutls are graphicaly shown in figure 6 . 


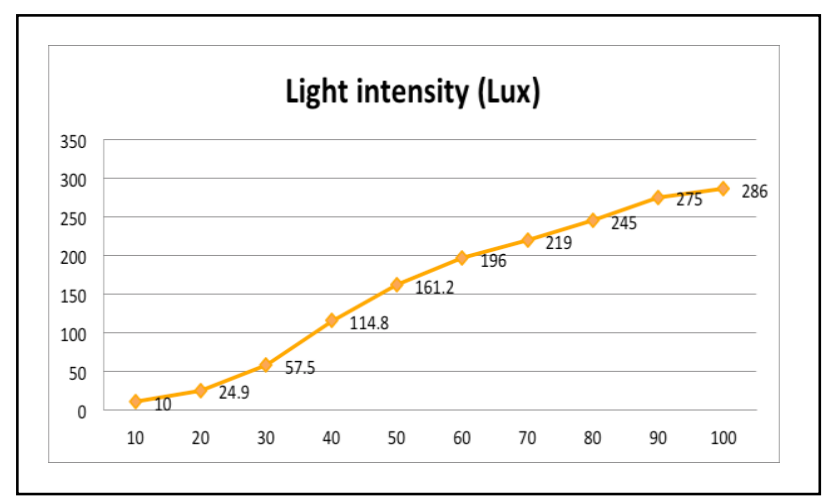

Fig. 6. Light intensity plot

\section{EDUCATIONAL USAGE OF THE DEVICE}

\subsection{Open hardware, open software}

The presented light dimmer device was not only developed to be used as a light dimmer, but also as an educational technology device. The complete project is based on the open software and open hardware movement.

An open hardware license is basically an agreement by the author(s) of the work that allows other people to use that work for free, without paying royalties, licensing fees as long as certain constraints are followed. The most common constraints are "attribution requirements", for example requiring anybody who uses the work to place the name of the original author(s) prominently on the final project. Another common constraint is "share-alike" that means that any derivative work must be released under a similar license.

Open source software is very often developed in public. Open-source software is the most prominent example of open-source development and often compared to (technically defined) user-generated content or (legally defined) open content movements [8].

The authors provide full documentation on the project at the following web page:

www.cset.com.hr/lightdimmer.

\subsection{Educational technology}

The presented device has a potential to be used as an educational technology tool to enhace the learning process in the education of electrical engineers. One of the key skills in electrical engineering is knowledge integration. That is, the possibility of the engineer to view the problem from different points of view, as well as the possibility to work in more than one field of specialization, in order to be able to comprehend the system that needs to be designed, repaired or otherway addressed.

The presented device offers practical knowledge gain in the following fields:

- optoelectronics (light propagation, infrared recievers and transmitters)

- digital electronics (microcontroller hardware and peripherals, microcontroller software, communication protocols)

- digital and analog circuits integration (digital to analog conversion and convertor types)

- analog electronics (signal amplification and filtering)
- power electronics (ballast systems, light intensity regulators)

- system integration knowledge

- printed circuit board design and manufacturing

- hands-on experience and prototype building

The end user (student) has the possibility to gain all the mentioned knowledge by designing, prototyping, building and analizing the proposed device.

\section{CONCLUSION}

The implemented universal IR light dimmer has met the criteria established in the introduction of this article. Testing results of light intensity regulations confermed the functionality of the implementd device. The avantage of such a device is its low price and flexibility. Also, it can be used as an educational technology tool to make knowledge integration easyer for future electrical engineers. Planned future work will include the development of a kit device easy to integrate and use in educational curricula.

\section{ACKNOWLEDGEMENTS}

The authors want to express gratitude to the Faculty of Engineering, Universty of Rijeka and the Croatian Society for Educational Technology for their support in the realization of this project.

\section{REFERENCES}

[1] http://www.epanorama.net/documents/lights/lightdimmer.html, (2000). , Accessed on: 2012-07-02

[2] http://www.dimming.org/Technical/detail/705.html, (2008)., Accessed on: 2012-06-27

[3] http://www.sbprojects.com/knowledge, (2011)., Accessed on: 2012-04-02

[4] Juergen Putzger. Decoding IR Remote controls, unpublished.

[5] Eric Maass (2001). How does a universal remote control work?, unpublished.

[6] Lamp Section, National Electrical Manufacturers Association (1998). Interaction of Infrared Controls And Electronic Compact Fluorescent Lamps, Report, unpublished

[7] http://www.ladyada.net/library/openhardware/whatisit.html, (2011)., Accessed on: 2012-07-05 